\title{
INVESTIGATIONS OF DEFECT CENTRES IN ALKALI HALIDES DOPED WITH TRIVALENT CATIONS. PART 2: THE NaCl:Cr ${ }^{3+}$ CRYSTALS
}

\author{
M. SUSZYŃSKa
}

W. Trzebiatowski Institute of Low Temperature and Structure Research Polish Academy of Sciences

Pl. Katedralny 1, 50-950 Wrocław, Poland

and R. CAPELletti

Physics Department, Parma University, 43100 Parma, Italy

Dedicated to Professor Dr. Julian Auleytner on the occasion of his 70th birthday

(Received July 9, 1992)

\begin{abstract}
On the basis of ITC studies, complemented by some spectroscopic and ionic conductivity measurements, it was stated that dielectric relaxations of the $\mathrm{NaCl}: \mathrm{Cr}^{3+}$ crystals are more complicated than those typical of $\mathrm{AH}: \mathrm{Me}^{2+}$ systems. The complexity of these phenomena concerns the number, shape and positions of the bands appearing in the ITC spectrum. The origin of the main peaks was discussed in terms of relaxations related with the chromium centres; substitutionally and off-center located $\mathrm{Cr}^{3+}$ ions, accompanied by two cation vacancies at geometrically different positions, and Cr clusters distributed around dislocations were considered.
\end{abstract}

PACS numbers: $77.40 .+\mathrm{i}$

\section{Introduction}

The present series of papers, devoted to alkali halides (AII) doped with "small" trivalent cations $\left(\mathrm{Me}^{3+}\right)$, began with characterization of bismuth centres in $\mathrm{NaCl}, \mathrm{KCl}$ and $\mathrm{RbCl}$ crystals (Part 1 [1]). Although some evidences for the presence of impurity-vacancy-vacancy (I.V.V.) dipoles were gathered, bismuth affinity to oxygen-like anions made the quantitative analysis of the obtained results difficult.

Similar to bismuth doped crystals the impurity related defects in chromium doped $\mathrm{NaCl}$ crystals were mainly studied by ionic thermocurrent (ITC) technique 
employed in an extended range of temperature for samples doped in a wide range of concentration, $c_{3+}$. In parallel, EPR spectra and the temperature dependence of ionic conductivity were also investigated. It should be noted that trivalent chromium is especially interesting for these studies because of laser and solar collector applications [2]. Moreover, for $\mathrm{NaCl}: \mathrm{Cr}^{3+}$ crystals the ratio of ionic radii $r_{3+} / r_{+}$is still smaller than for the bismuth doped $\mathrm{AH}$, and still more similar to doped silver halides with the structure of sodium chloride. For this reason, the results obtained are critically compared with those presented by Ulrici [3] and Kunze et al. [4] for silver halides doped with trivalent chromium; the most characteristic features of ITC spectra typical of AH: $\mathrm{Me}^{2+}$ systems with $r_{2+} \geq r_{+}$were also shortly discussed.

\section{Crystals and techniques used}

\subsection{Basic characteristics of samples}

Single crystals of $\mathrm{NaCl}$ containing trivalent chromium ions were grown by a modified Bridgman method [5] at the Crystal Growth Laboratory, Institute of Physics, CSAV, Prague by Dr M. Lebl.

The nominal concentration of the dopant ranged between 10 and 2000 mole ppm. The chromium content was determined by the atomic absorption spectrometry (AAS), whereby a Perkin-Elmer spectrometer was used; for the numerical data see Table I.

TABLE I

Characterization of crystals.

\begin{tabular}{c|r|c|ll}
\hline \hline \multirow{2}{*}{ Mark } & \multicolumn{2}{|c|}{ Dopant [ppm] } & \multirow{2}{*}{ Remarks } \\
\cline { 2 - 3 } & Melt & AAS/OA & \\
\hline F.1 & 10 & $4.5 /-$ & 1. & $r(\mathrm{Cr}: 3+)=0.63 \AA$, \\
F.2 & 30 & $-/ 0.38$ & & $r(\mathrm{Na}:+)=0.98 \AA$, \\
F.5 & 300 & $-/ 0.38$ & & $r=0.63 / 0.98=0.64$. \\
F.6 & 500 & $14 / 1.04$ & 2. & $\mathrm{Cr}(3+)$ and $\mathrm{Cr}(+)$ \\
F.7 & 800 & $-/ 1.60$ & & ions are paramagnetic. \\
F.8 & 1000 & $-/ 1.72$ & 3. & OA gives the absorption \\
F.9 & 2000 & $40 / 2.80$ & & coefficient at $\lambda \cong 234 \mathrm{~nm}$.
\end{tabular}

The major part of experiments was performed for as received (AR) about 15 years old crystals. In some instances the effect of high-temperature treatment was studied. After 30 minutes of annealing at $873 \mathrm{~K}$ the sample was dropped onto a copper plate at room temperature (RT); this procedure was considered as determining the solution treated (ST) state of samples. 


\subsection{ITC and other measurement techniques}

If not otherwise stated, the ionic thermocurrent (ITC) measurements were performed under conditions usually applied for detection of the impurity-vacancy (I.V.) dipoles in alkali halides doped with divalent cations [6]. Additionally to the low-temperature phenomena, polarizations appearing above RT were registered. The effect of plastic deformation (uniaxial compression in a self-made device) upon the ITC spectra was detected for an arrangement described previously [7].

The valency of the dopant was systematically verified by taking the EPR spectra at RT and/or liquid nitrogen temperature (LNT) with the VARIAN-V-4502-12 spectrometer operating with $100 \mathrm{kHz}$ field modulation. The orientation of samples was achieved by means of a goniometer, and for the purposes of this work the samples were rotated around angle $\Theta$ between the magnetic field and the main crystal axes.

The temperature dependence of ionic conductivity was measured in the same way as it has been described in Part 1 [1].

\section{Results and discussion}

\subsection{Ionic conductivity}

Figure 1 shows the conductivity $\sigma$ versus inversion of temperature $1 / T$ for AR samples doped with various amounts of chromium.

Similar to bismuth doped $\mathrm{NaCl}$ crystals (Part 1) the temperature dependence of $\sigma$ displays two characteristic regions, the extrinsic and association one. Hence, the conductivity plots of slightly doped samples are explainable in terms of substitutionally incorporated $\mathrm{Cr}^{3+}$ ions for which the charge excess is compensated by cation vacancies. With increasing $c_{3+}$ the association of vacancies occurs at temperatures at which the intrinsic conductivity takes place. Similar to the bismuth doped alkali halides [1], the number of cation vacancies, induced by each $\mathrm{Cr}^{3+}$ ion, is larger than one. The migration and association energies, obtained from the $\lg (\sigma T)$ vs. $1 / T$ plot for AR samples, are listed in Table II; for comparison, experimental data for $\mathrm{NaCl}$ crystals doped with other transition metal ions are also presented. The values obtained are similar to each other, whence the effect of electric charge seems rather poor, at least for the migration. This result is in contrast with the classical theory by Tosi and Doyama [10] in which the charge as well as size of the dopant are strongly involved.

\subsection{EPR spectra}

For heavily doped samples the EPR spectra detected at RT are composed of different lines, whose number increases with decreasing temperature. The large number of lines is probably the consequence of several overlapping spectra related with several paramagnetic centres, whose $g$-factor takes an average value of 2.0036 .

To obtain information about the symmetry and/or the relative number of centres, preliminary studies of the signal anisotropy were undertaken. The crystals 


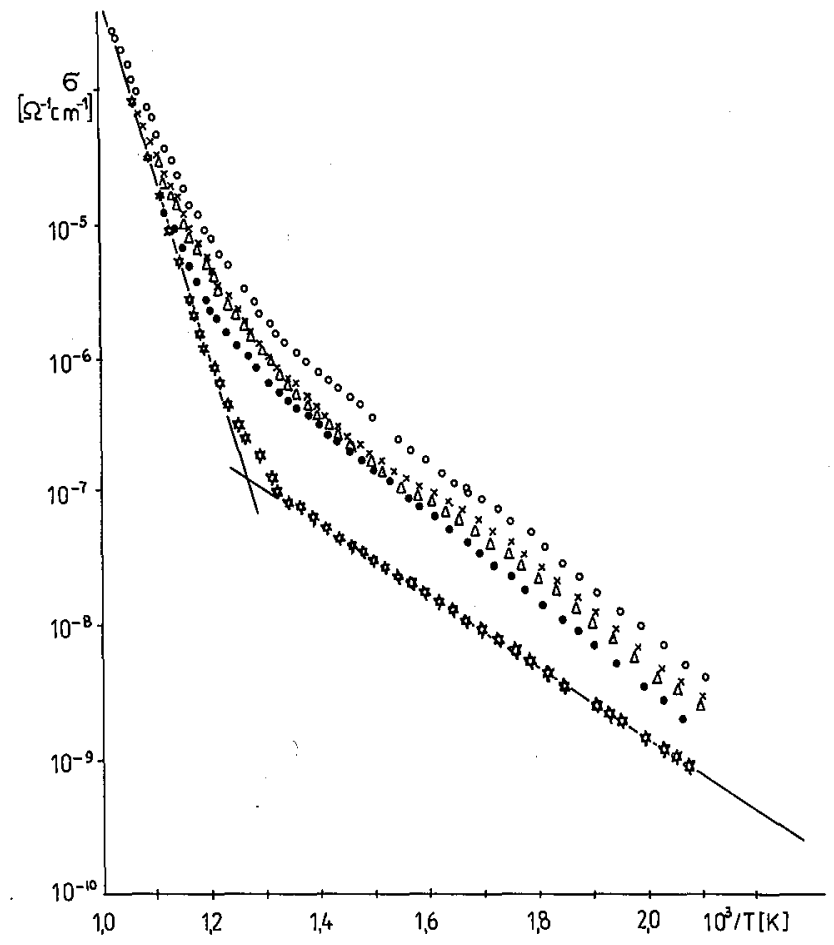

Fig. 1. Temperature dependence of the electric conductivity of AR samples doped with $30(\bullet), 300(\triangle), 800(\times)$ and $2000(0)$ mole ppm of $\operatorname{Cr}(3+)$; curve (*) shows data characteristic of nominally pure $\mathrm{NaCl}$ crystals.

were rotated in (100) and (110) planes by 2.5 angular degree. Figure 2 exemplifies the results obtained by rotating the F.3 crystal about a $\langle 100\rangle$ direction. Although

TABLE II Activation energies of $\mathrm{Cr}(3+)$ ions compared with those characteristic of some transition metal ions incorporated substitutionally in $\mathrm{NaCl}$ crystals.

\begin{tabular}{r|l|l|c}
\hline \multirow{2}{*}{ Ion } & \multicolumn{2}{|c|}{ Energy of } & \multirow{2}{*}{ Refs. } \\
\cline { 2 - 3 } & association & migration & \\
\hline $\mathrm{Mn}(2+)$ & $0.3 \div 0.6$ & $0.8 \div 0.95$ & {$[8]$} \\
$\mathrm{Ni}(2+)$ & $0.32,0.62$ & 0.75 & {$[9]$} \\
$\mathrm{Co}(2+)$ & 0.3 & 0.75 & {$[9]$} \\
$\mathrm{V}(2+)$ & 0.43 & 0.7 & {$[9]$} \\
$\mathrm{Cr}(3+)$ & 0.68 & 0.78 & here
\end{tabular}




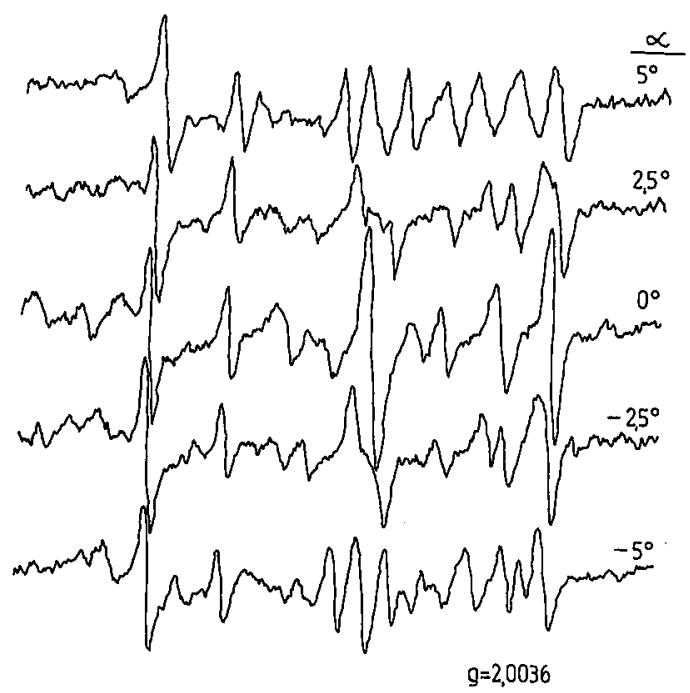

Fig. 2. Room-temperature EPR spectra of the F.3 sample showed for various values of $\alpha$ - the angle between the [100] direction and the magnetic field $H$; sweep frequency $\cong 100 \mathrm{~Hz}$.

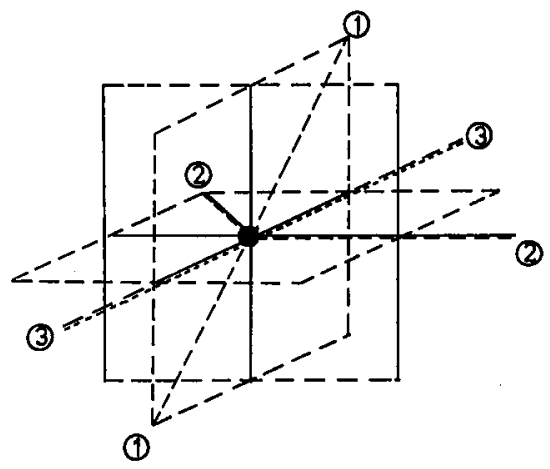

Fig. 3. $\mathrm{Cr}^{3+}$ in the $\mathrm{NaCl}$ matrix, associated with two vacancies in the $\mathrm{nn}$ and $\mathrm{nnn}$ positions. The symmetries $D_{2 h}, C_{s}$ and $D_{4 h}$ are represented by vacancy pairs labelled 1,2 and 3 respectively; black point marks the $\operatorname{Cr}(3+)$ ion position.

the observed spectra were not analysed in sufficient details to fit an appropriate IIamiltonian, the angular dependence of the position of some spectral lines can be related with centres of a twofold and a fourfold symmetry. These symmetries are characteristic of defects composed of the impurity ions and cation vacancies in the nearest neighbour (nn) and next nearest neighbour (nnn) position, respectively. Figure 3 shows the most probable configurations of I.V.V. dipoles. The symmetry of these centres could be as follows: 
1. $D_{2 h}$ with two vacancies in the $\mathrm{nn}$ position to $\mathrm{Cr}^{3+}$ and in transposition to each other;

2. $C_{s}$ - which arises when one vacancy is in the nn site and the other one in the nnn position, whereby both dipole axes are in the same [100] plane; and

3. $D_{4 h}$ - when both vacancies occupy the nnn sites in the transposition to each other.

Since two cation vacancies repel each other by the Coulombic interactions, the $D_{2 h}$ and other configurations with both vacancies in the nearest neighbour sites to each other are less probable. The relative number of various dipoles depends on the dopant concentration, thermal pretreatment and plastic deformation of samples; more detailed data will be reported shortly.

\subsection{ITC data}

\subsubsection{The results obtained}

The depolarization currents of $\mathrm{NaCl}: \mathrm{Cr}(3+)$ crystals show a peak near $226 \mathrm{~K}$ (A.1) accompanied by another one at $206 \mathrm{~K}(A .2)$ and a broad high-temperature band $(C)$. For the low-temperature range the effects of polarization conditions are shown in Fig. 4 for AR (curves 1 and 2) and ST samples of the F.6 crystal (the remaining curves). Figure 5 shows the most characteristic features of the lowand high-temperature bands. On assuming that the temperature dependence of the relaxation time related with the low-temperature band is of Arrhenius type, the activation parameters for reorientation jumps may be calculated. Because of some experimental limitations, the activation energy $E$ was evaluated according to Chen's approximations [11], whereas the frequency factor $\tau_{0}$ was calculated from the following equation describing the condition for the band maximum:

$$
T_{\mathrm{m}}=\left[(E / k) b \tau\left(T_{\mathrm{m}}\right)\right]^{1 / 2} .
$$

For the Arrhenius-type jumps:

$$
\tau=\tau_{0} \exp \left(-E / k T_{\mathrm{m}}\right)
$$

and

$$
\tau_{0}=T_{\mathrm{m}}^{2} k / E b \exp \left(E / k T_{\mathrm{m}}\right)
$$

with $b$ and $k$ denoting the heating rate (in our experiments equal to $0.1 \mathrm{~K} \mathrm{~s}^{-1}$ ) and Boltzmann constant, respectively. Table III lists the numerical values of $E$ and $\tau_{0}$, and Fig. 6 shows the reorientation energy of Cr dipoles compared with those characteristic of divalent impurities in $\mathrm{NaCl}$ crystals as function of their ionic radius.

The main features of the $C$ band may be summarized as follows:

1. The amplitude $H^{C}$ and peak position $T_{\mathrm{m}}^{C}$ are increasing functions of the thickness of samples $X$.

2. For fixed $X$ the $T_{\mathrm{m}}^{C}$ value depends on the polarization conditions, i.e. the polarizations related with this band should be described by a distribution of relaxation times, being meaningful for shape- and/or size distribution of particles segregated near dislocations, cp. Part 1 [1]. 


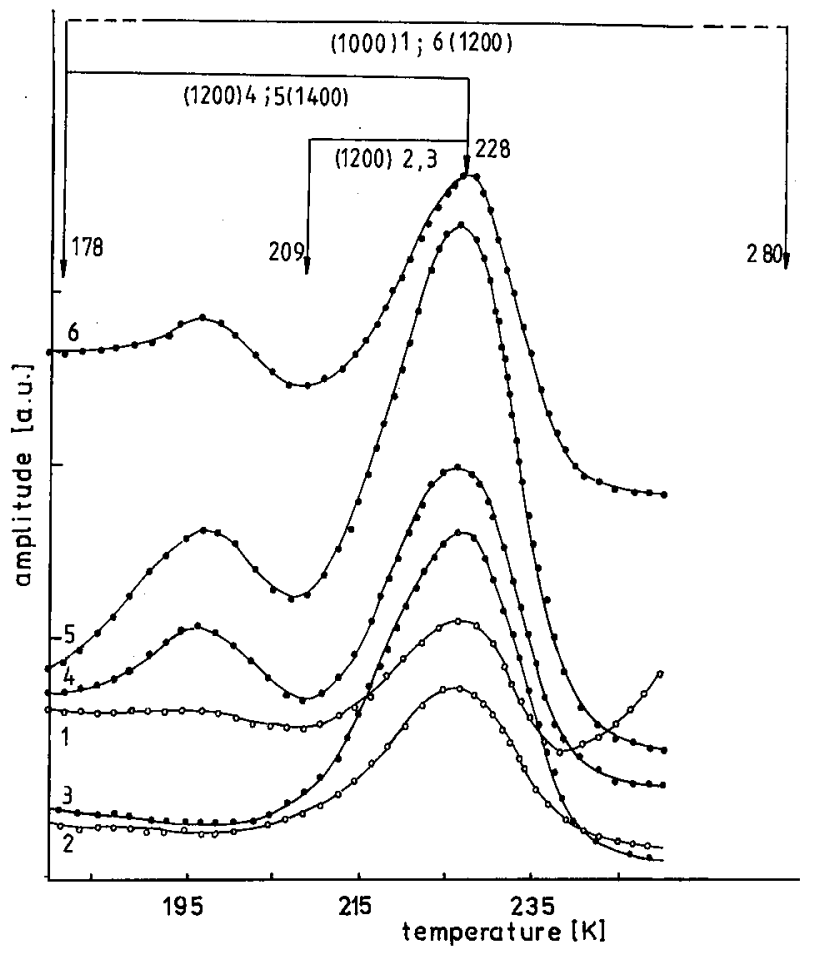

Fig. 4. ITC spectra characteristic of the F. 6 sample; the effect of polarization conditions for AR (curves 1,2 ) and ST samples (remaining curves) is shown.
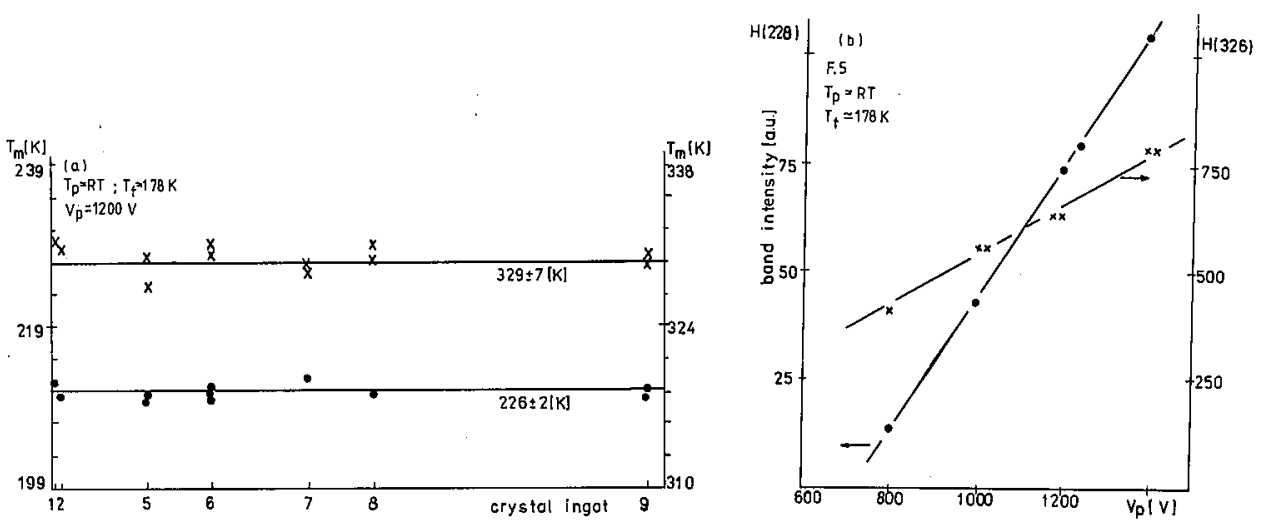

Fig. 5. Characteristic peak positions for the crystals studied (a) and the dependence of peak amplitudes on the electric field applied (b). 


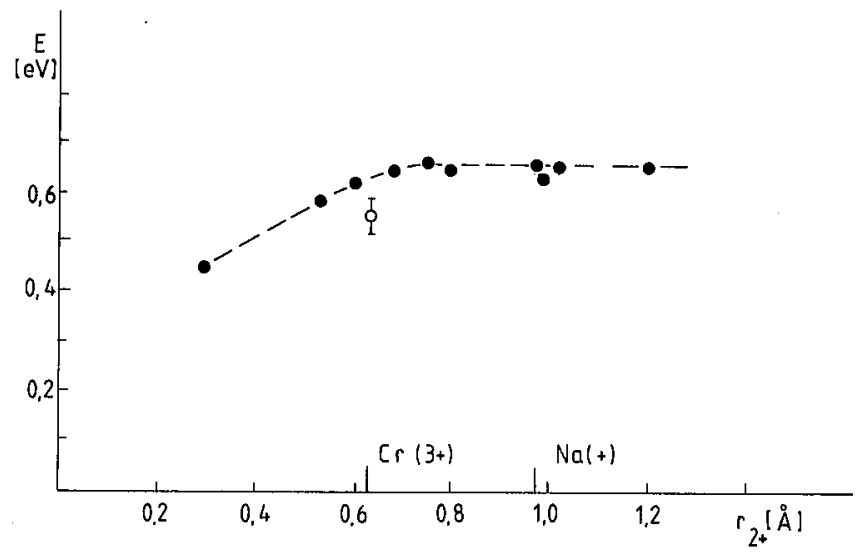

Fig. 6. Reorientation activation energy of I.V. dipoles in $\mathrm{NaCl}$ crystals vs. ionic radius of divalent impurities according to [12]; the average values obtained for $\mathrm{NaCl}: \mathrm{Cr}(3+)$ are also presented.

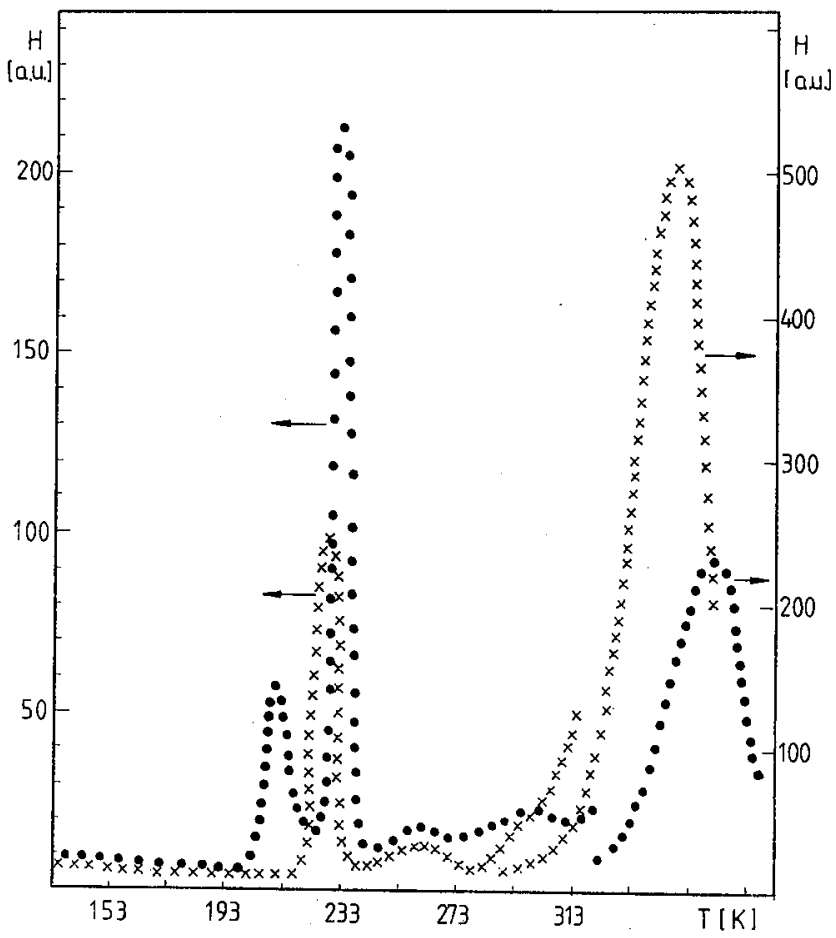

Fig. 7. Effect of plastic deformation upon ITC spectrum of the F.2 sample (•); for comparison the spectrum of AR sample is also presented $(\times) . T_{\mathrm{p}} \cong \mathrm{RT}, T_{\mathrm{f}}=178 \mathrm{~K}$, $V_{\mathrm{p}}=1460 \mathrm{~V}$. 
3. Similar to the dipolar bands, the frozen-in polarizations (represented by $\left.H^{C}\right)$ are linear with $E_{\mathrm{p}}\left(=V_{\mathrm{p}} / X\right)$ up to about $3 \times 10^{3} \mathrm{~V} \mathrm{~cm}^{-1}$.

4. Few percent of plastic deformation $(\varepsilon)$ induces strong decrease in $H^{C}$, distinct shift of $T_{\mathrm{m}}^{C}$ towards higher values and drastic increase in the bandwidth, see Fig. 7, where data for $\varepsilon=0 \%$ are shown for the sake of comparison.

TABLE III

Relaxation parameters characterizing the low-temperat ure ITC bands for plate-shaped and parallelepipeds in as received, aged after solution treatment and deformed samples of $\mathrm{NaCl}$ containing various amounts of $\mathrm{Cr}(3+)$ ions.

\begin{tabular}{c|c|c|c|c|c}
\hline \hline Mark & F.6.6 & F.7.1 & F.8.1 & F.9.8 \\
Shape & parall. & $\begin{array}{c}\text { plate } \\
\text { parall. }\end{array}$ & $\begin{array}{c}\text { F.9.8 } \\
\text { parall. }\end{array}$ \\
Treatment & AR + 13.3\% & ST + aged/RT & ST + aged/ST & ST + 4.56\% & ST + 4.56\% \\
$T_{\mathrm{m}}[\mathrm{K}]$ & 211.5 & 211 & 213 & 206 & 182 \\
$\mu=\frac{T_{2}-T_{\mathrm{m}}}{T_{2}-T_{1}}$ & 0.40 & 0.44 & 0.42 & 0.44 & 0.50 \\
$E_{\alpha}[\mathrm{eV}]$ & 0.56 & 0.52 & 0.56 & 0.56 & 0.49 \\
$E_{\delta}[\mathrm{eV}]$ & 0.50 & 0.47 & 0.55 & 0.51 & 0.35 \\
$E_{\omega}[\mathrm{eV}]$ & 0.54 & 0.50 & 0.56 & 0.54 & 0.42 \\
$\langle E\rangle[\mathrm{eV}]$ & 0.53 & 0.50 & 0.56 & 0.54 & 0.42 \\
$\tau_{0 \alpha}[\mathrm{s}]$ & $4.16 \times 10^{-12}$ & $2.53 \times 10^{-11}$ & $3.16 \times 10^{-12}$ & $1.54 \times 10^{-12}$ & $1.69 \times 10^{-12}$ \\
$\tau_{0 \delta}[\mathrm{s}]$ & $8.52 \times 10^{-11}$ & $5.50 \times 10^{-10}$ & $8.70 \times 10^{-12}$ & $2.53 \times 10^{-11}$ & $1.91 \times 10^{-8}$ \\
$\tau_{0 \omega}[\mathrm{s}]$ & $1.23 \times 10^{-11}$ & $8.37 \times 10^{-11}$ & $3.70 \times 10^{-12}$ & $4.15 \times 10^{-12}$ & $1.83 \times 10^{-10}$ \\
$\langle\tau\rangle[\mathrm{s}]$ & $1.72 \times 10^{-11}$ & $1.05 \times 10^{-10}$ & $4.69 \times 10^{-12}$ & $5.55 \times 10^{-12}$ & $1.83 \times 10^{-10}$
\end{tabular}

\subsubsection{Considerations of the relaxation phenomena}

The low-temperature band $A .1$ is located in the temperature range where the dipolar band occurs for $\mathrm{AH}$ crystals doped with small $\mathrm{Me}^{2+}$ ions. Although the amplitude of this band is linearly dependent on the polarization voltage, its shape is slightly different from the nonsymmetrical one characteristic of I.V. dipoles. The broadening observed with increasing $c_{3+}$ probably reflects various geometrical configurations of coexisting I.V.V. dipoles. If so, the $\alpha$-approach, practically insensitive to the kinetics order, should be considered as the best estimation of the activation energy value. The increase in $c_{3+}$ induces not only the broadening of the main low-temperature band but also the appearance of a second band (A.2) positioned below the main one. This phenomenon resembles the situation in silver halides doped with trivalent chromium for which the presence of nn and nnn types of dipoles was suggested [4]. It should be noted, however, that for $\mathrm{NaCl}: \mathrm{Cr}^{3+}$ crystals the $A .2$ band appears also in solution treated and plastically deformed samples. These "agents" induce lattice distortion around the $\mathrm{Cr}^{3+}$ ion, which might be interpreted as equivalent to an off-centre position. Since the dipolar moment of such defects is smaller than for the centrally located cations, see [13], the amplitude of both low-temperature bands is not simply proportional to the dopant concentration.

Although the behaviour of the high-temperature part of the spectrum is explainable in terms of the Maxwell-Wagner polarizations, some features of these 
bands are distinctly different from those detected for $\mathrm{AH}: \mathrm{Me}^{2+}$ systems [7]. It seems reasonably to assume that in the case of small trivalent cations, both the extended and compressed regions near edge dislocations are accessible for the impurities, and instead of the formation of a classical Cottrell atmosphere (typical of $\mathrm{AH}: \mathrm{Me}^{2+}$ systems) an "unlimited" number of cations may be segregated here, probably in the form of aggregates and/or precipitates.

\section{Concluding remarks}

The main conclusion of the EPR studies - that in $\mathrm{NaCl}: \mathrm{Cr}^{3+}$ crystals may coexist centres of different configuration - was confirmed by the composed character of ITC bands. Also the suggestion from conductivity data - that more than one cation vacancy accompanies the substitutionally positioned $\mathrm{Cr}^{3+}$ ion - found further support in the geometry of dipolar centres related with the low-temperature dielectric relaxations.

Comparing the features of the polarization phenomena detected with those typical of AH:Me ${ }^{2+}$ crystals with $r_{2+} \geq r_{+}$, some differences were found. They concern the number, shape and position of the relaxation bands appearing in the low-temperature range. Also the high-temperature bands react differently to plastic deformation and thermal pretreatment as well. In a given sense the spectra appearing in $\mathrm{NaCl}: \mathrm{Cr}^{3+}$ also differ from those recently described for $\mathrm{LiF}: \mathrm{Ti}^{3+}$ crystals (with $r_{3+} \geq r_{+}$) [14]. On the contrary, some similarities with the behaviour of the off-centre positioned $\mathrm{Be}^{2+}$ ions in $\mathrm{NaCl}$ and $\mathrm{KCl}$ crystals [13] and the substitutionally located $\mathrm{Cr}^{3+}$ ions in silver halides [4] were detected. Hence, it seems reasonable to suppose that the size of the impurity ion plays an important role in determining the main features of the polarizations registered by the sensitive ITC technique. Taking into account the conductivity data, this means that the second charge compensating cation vacancy is probably at a distance different from the considered $\mathrm{nn}$ and nnn ones. The evidences could be attained by studies of the EPR anisotropy. Moreover, the distribution of cation vacancies around $\mathrm{Cr}^{3+}$ ions and the electrostatic interactions between them should influence the degeneracy and activity of electronic and vibrational transitions as well. Hence, also spectroscopic investigations could be the basis for final determination of the structure of defects investigated.

\section{Acknowledgments}

The authors would like to express their great appreciation to Dr. M. Lebl for the supply of crystals. They are also grateful to Mrs. M. Szmida for technical assistance.

\section{References}

[1] M. Suszyńska, R. Capelletti, Acta Phys. Pol. A 83, 25 (1993).

[2] L.M. Andrews, J. Lumin. 24/25, 877 (1981).

[3] W. Ulrici, Phys. Status Solidi 27, 489 (1968). 
[4] K. Kunze, P. Müller, Phys. Status Solidi 38, 271 (1970).

[5] R. Voszka, J. Tarjan, L. Berkes, J. Krajsovszky, Krist. Technik 1, 423 (1966).

[6] C. Bucci, R. Fieschi, G. Guidi, Phys. Rev. 148, 816 (1966).

[7] M. Suszyńska, R. Capelletti, Cryst. Res. Technol. 19, 1489 (1984).

[8] F. Beniere, R. Rokbani, J. Phys. Chem. Solids 36, 1151 (1975).

[9] M. Hartmanova, M. Lebl, E. Mariani, Phys. Status Solidi A 31, k 85 (1975).

[10] M.P. Tosi, M. Doyama, Phys. Rev. 151, 642 (1966).

[11] R. Chen, J. Mater. Sci. 11, 1521 (1976).

[12] R. Capelletti, in: Defects in Solids, Eds. A.V. Chadwick, M. Terenzi, Plenum, London 1986, p. 407.

[13] C. Bucci, Phys. Rev. 164, 1200 (1967).

[14] R. Capelletti, M.G. Bridelli, M. Friggeri, G. Ruani, in: Proc. 5th Symp. Electrets, IEEE, Heidelberg (Germany) 1985, IEEE, New York 1985, p. 294. 